site.

Keywords: DNA, photooxidation, time-resolved spectroscopy

\section{MS12-O4 Racemic DNA Crystallography:} advantages and applications

Pradeep K. Mandal ${ }^{1,2}$, Gavin W. Collie ${ }^{1,2}$, Suresh C. Srivastava ${ }^{3}$, Brice Kauffmann ${ }^{4,5,6}$, Ivan Huc ${ }^{1,2}$

1. Université de Bordeaux, CBMN (UMR5248), Pessac 33600, France

2. CNRS, CBMN (UMR5248), Pessac 33600, France

3. ChemGenes Corporation, 33 Industrial Way, Wilmington, MA 01887, USA

4. CNRS, Institut Européen de Chimie et Biologie (UMS3033), Pessac 33600, France

5. INSERM, Institut Européen de Chimie et Biologie (US001), Pessac 33600, France

6. Université de Bordeaux, Institut Européen de Chimie et Biologie (UMS3033), Pessac 33600, France

email: pk.mandal@iecb.u-bordeaux.fr

The use of racemic mixtures of naturally chiral macromolecules such as proteins and nucleic acids can significantly increase the chances of crystallization. Racemates allow molecular contacts to be formed in a greater number of ways and give access to centrosymmetric space groups. The benefits of applying racemic crystallographic methods to proteins are considerable (1). Curiously, however, racemic DNA crystallography had not been established despite the commercial availability of L- deoxyribo-oligonucleotides. We present a study into racemic DNA crystallography over a diverse range of sequences and folded conformations including duplexes, four-way Junctions and G-quadruplexes. This study revealed a strong propensity of racemic DNA mixtures to form racemic crystals (2). The crystals obtained from racemic mixtures were invariably achiral, showing a preference for the space group P1(bar) and the structures determined were nearly identical to those determined from classical D-enantiopure solutions (2). Following this proof-of-concept, we evaluated the racemic crystallographic approach for the crystallization of an intractable, biologically relevant DNA sequence - that of the Pribnow box consensus sequence 5'-TATAAT-3' in a duplex with its complementary sequence. Four high-quality crystal structures were determined with resolutions in the range of $1.65-2.3 \AA$, providing insights into the racemic crystallization process as well as structural details of this biologically relevant sequence (3). The racemic crystallization technique provides an attractive alternate to conventional crystallization using D-enantiopure solutions alone. It shows potential for the study of native DNA sequences beyond the classical model sequences, and thus may be of interest to those investigating structural aspects of fundamental biological processes and structure-based DNA-directed drug development programs. This work was supported by the European Union's Seventh Framework Programme through the European Research Council (Grant Agreement No. ERC-2012-AdG-320892, post-doctoral fellowship to PKM).

References:

(1) Yeates, T. O. \& Kent, S. B. H. (2011). Annu. Rev. Biophys., 41, 41-61.

(2) Mandal, P. K., Collie, G. W., Kauffmann, B. \& Huc, I. (2014). Angew. Chem. Int. Ed., 53, 14424-14427.

(3) Mandal, P. K., Collie, G. W., Srivastava, S. C., Kauffmann, B. \& Huc, I. (submitted). 


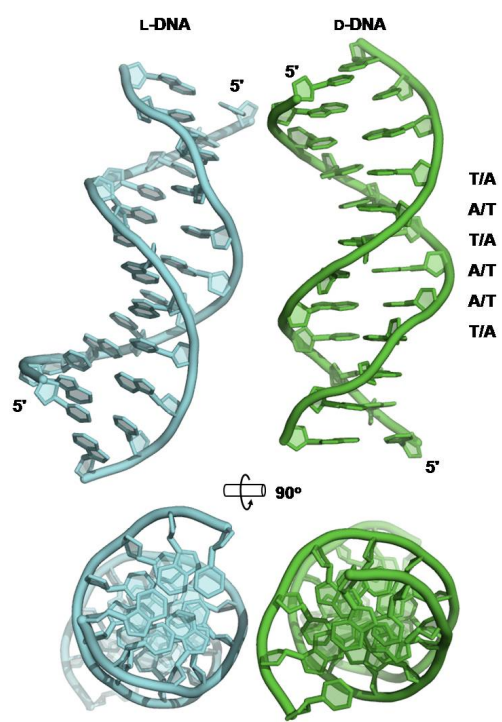

Figure 1. Crystal structure (in space group $P$ bca, resolution 1.65 A) of a DNA duplex containing the Pribnow box consensus promoter sequence 5'-TATAAT-3' with its complementary strand. Right: asymmetric unit, containing one complete D-DNA duplex (green). Left: L-DNA symmetry mate (cyan).

Keywords: Racemic crystallography, DNA, X-ray diffraction, DNA crystallography
MS12-O5 An all-in-one lanthanide complex to overcome the two major bottlenecks in protein crystallography.

Sylvain Engilberge ${ }^{1}$, Louise Lassalle ${ }^{1}$, Sebastiano Di Pietro ${ }^{2}$, François Riobé ${ }^{2}$, Olivier Maury ${ }^{2}$, Eric Girard ${ }^{1}$

1. Institut de Biologie Structurale, UMR5075 CEA-CNRS-UJF, EPN Campus, 71 avenue des Martyrs, 38044 Grenoble Cedex 9 2. UMR5182 CNRS-ENS Lyon-Université Lyon 1, 46 allée d'Italie, 69364, Lyon cedex

email: sylvain.engilberge@ibs.fr

Protein crystallography remains the major technique for protein structure determination. Among 117438 structures deposited on the protein data bank (April 2016), $89 \%$ had been solved using X-ray crystallography. However, protein crystallography is still limited by two major bottlenecks: Obtaining protein crystals and solving the phase problem.

Since 2000, we developed lanthanide complexes for structure determination of macromolecules, exploiting the high-phasing power of lanthanide elements [1-7]. Recently, we produced a new complex with luminescent properties. Unexpectedly, this lanthanide complex ([Ln]) showed nucleant properties making it the first nucleant, luminescent and phasing agent.

To demonstrate the effects of this new molecule, we selected 8 proteins with different molecular weights and different oligomeric states. Among these 8 proteins, two were of unknown structure including one with an unknown fold.

We performed comparative crystallization screening at the HTX lab crystallization plate form (EMBL Grenoble) with and without [Ln]. We showed that [Ln] significantly increases the number of crystal hits and more importantly, it provides unique crystallization conditions. In many cases the incorporation of [Ln] significantly increases the crystal quality as shown in Figure 1.

The luminescence properties of [ $\mathrm{Ln}]$ have been used for crystals detection and may be exploited for crystal centering on synchrotron beam line.

The structures of the 8 proteins have been determined using anomalous based phasing methods.

In conclusion, this new all-in-one lanthanide complex allows to overcome the two majors bottlenecks in protein crystallography.

[1] E. Girard et al. Acta Cryst D, 59, (2003), 118.[2] E. Girard et al. Acta Cryst D, 59, (2003), 1914.[3] R. Talon et al. J. Synch. Rad., 18, (2011), 74. [4] G. Pompidor et al. Acta Cryst D, 66, (2010), 762.[5] G. Pompidor et al. Angew. Chem. Int. Ed., 47, (2008), 3388. [6] E. Dumont et al. Phys. ChemChemPhys., 15, (2013), 18235. [7] A. D'Aléo et al. ChemPhysChem., 8, (2007), 2125.

We acknowledge financial support from the Agence Nationale de la Recherche (ANR-13-BS07-0007-02 Ln23). 